\title{
Concomitant Face/Upper Extremity Allotransplantation
}

\author{
David A. Leonard • Melissa Mastroianni • \\ Kumaran Shanmugarajah • Curtis L. Cetrulo Jr.
}

Published online: 22 December 2012

(C) Springer Science + Business Media New York 2012

\begin{abstract}
Concomitant vascularized composite tissue allotransplantation (VCA) offers single stage restoration of complex anatomical units in a manner that would be difficult, if not impossible to accomplish using conventional reconstructive techniques. However, such extensive and complex transplant procedures have considerable inherent risk. Two cases of concomitant face and hand transplantation have been performed to date, both with significant early complications leading to significant morbidity, and in one case, mortality. These cases highlight some of the unknowns in both perioperative and immunological management that must be addressed if this complex subset of VCA procedures is to be more widely offered. Only once these acute challenges can be reliably managed will focus turn to rehabilitation and long-term outcomes. If these issues can be overcome, concomitant face and upper
\end{abstract}

D. A. Leonard - M. Mastroianni - K. Shanmugarajah ·

C. L. Cetrulo Jr. ( $\square)$

Reconstructive Transplantation Service, Division of Plastic and Reconstructive Surgery, Massachusetts General Hospital, Boston, MA 02114, USA

e-mail: ccetrulo@partners.org

D. A. Leonard

e-mail: david.leonard@tbrc.mgh.harvard.edu

M. Mastroianni

e-mail: melissa.mastroianni@tbrc.mgh.harvard.edu

K. Shanmugarajah

e-mail: kumaran.shanmugarajah@tbrc.mgh.harvard.edu

D. A. Leonard - M. Mastroianni - K. Shanmugarajah ·

C. L. Cetrulo Jr.

Transplantation Biology Research Center, MGH-East,

Building 149, 13th Street, Boston, MA 02129, USA extremity transplantation could offer patients who have suffered severe, complex injuries unprecedented options for restoration of function and form.

Keywords Vascularized composite allotransplantation . Face transplant . Upper extremity transplant . Upper extremity allotransplantation · Concomitant transplantation - Functional outcomes · Patient selection

\section{Introduction}

Vascularized composite tissue allotransplantation (VCA) is now considered a feasible reconstructive option for patients with severe injury and tissue loss. A variety of VCAs, including the face, upper extremity, lower extremity, abdominal wall, larynx, and knee have been performed worldwide. Currently 23 face transplants and over 80 upper extremity transplants have been reported $[1,2 \bullet, 3 \bullet \cdot]$. Blasts, burns and animal attacks are among the traumatic mechanisms which may result in extensive injuries across multiple anatomical areas. Such injuries and the potential to improve on the outcomes of conventional reconstructive procedures in many of these cases have led to the emergence of concomitant transplantation of more than one VCA as a reconstructive solution. Due to the surgical challenge and the associated risk of morbidity and mortality, concomitant transplantation is considered the most complex type of VCA (Table 1) [4•]. Simultaneous transplantation of face and upper extremity has, to date, been attempted only twice $[5 \cdot \bullet, 6 \bullet \cdot]$. This review will examine these cases, evaluate the outcomes, identify questions arising from these cases, and offer some potential suggestions for future directions in this field. 
Table 1 The Gordon VCA classification (Adapted from Siemionow et al. [20])

\begin{tabular}{|c|c|c|c|}
\hline Type & Complexity & $\begin{array}{l}\text { Examples of } \\
\text { transplantable tissues }\end{array}$ & Characteristics \\
\hline I & Low & $\begin{array}{l}\text { Flexor tendon, tongue, } \\
\text { uterus, vascularized } \\
\text { nerve }\end{array}$ & $\begin{array}{l}\text { No skin } \\
\text { Low antigenicity }\end{array}$ \\
\hline II & Moderate & $\begin{array}{l}\text { Abdominal wall, ear, } \\
\text { genitalia, larynx, } \\
\text { scalp, trachea, } \\
\text { vascularized knee } \\
\text { joint }\end{array}$ & $\begin{array}{l}\text { May contain skin } \\
\text { Absent or minimal } \\
\text { rehabilitation }\end{array}$ \\
\hline III & High & Upper extremity, face & $\begin{array}{l}\text { Requires a } \\
\text { multidisciplinary } \\
\text { team and complex } \\
\text { rehabilitation } \\
\text { Significant } \\
\text { psychosocial } \\
\text { obstacles } \\
\text { Complex cortical } \\
\text { reorganization } \\
\text { occurs }\end{array}$ \\
\hline IV & Maximum & Concomitant VCA & $\begin{array}{l}\text { High mortality risk } \\
\text { Extremely difficult } \\
\text { rehabilitation } \\
\text { Unknown functional } \\
\text { outcomes }\end{array}$ \\
\hline
\end{tabular}

\section{Clinical Cases}

The first concomitant face and hand transplant was performed in Paris in April 2009 [5••]. The patient was a 37-year-old man who had sustained a burn injury involving $80 \%$ of his total body surface area. All 10 fingers had been amputated and his upper face and skull had been reconstructed with a free omental flap and split thickness skin grafts. He received a partial myocutaneous facial allograft and bilateral below elbow transplants. This patient was diagnosed with multi-drug resistant pseudomonas aeroguinosa infection of face and left hand allografts on postoperative day 15 . The patient subsequently developed areas of necrosis affecting the facial allograft tissue, requiring repeated surgical excisions and antibiotic therapy. On postoperative day 33 , the patient suffered airway obstruction and cardio-respiratory arrest during treatment of septic hemorrhage. Despite 5 weeks of intensive care, the patient died 65 days post transplant [5••].

The second concomitant face and hand transplant was performed in Boston in May 2011 [6••]. The recipient was a 57-year-old female who sustained extensive injury to her face and hands in a chimpanzee attack. This patient received an extensive osteomyocutaneous facial allograft, and bilateral hand transplants at distal forearm level. On post-operative day 2, the patient developed aspiration pneumonia which progressed to septic shock, ultimately resulting in irreversible ischemic damage to both transplanted hands. The upper limb allografts required amputation on post-operative day 5 . The patient was transferred out of the ICU on post-operative day 18 and discharged from the hospital on post-operative day 38 [6••].

\section{Surgical Challenges}

The heterogeneous nature of injuries experienced by patients considered for face and hand transplantation necessitates significant variation in the extent of VCA tissue harvested. Facial allografts have varied considerably in terms of the percentage facial area transplanted, and have included both myocutaneous flaps composed of the skin and muscles of facial expression, and osteomyocutaneous flaps including significant parts of the mid- or lower facial skeleton $[1,5 \bullet \bullet, 6 \bullet \bullet, 7,8 \bullet, 9-15]$. Similarly, both unilateral and bilateral hand transplants have been performed at different levels between the wrist and shoulder $[2 \bullet, 3 \bullet \bullet]$. To date, there have been no reported losses of isolated facial or upper extremity allografts (whether unilateral or bilateral) in the acute phase as a result of surgical complications and, uniquely for vascularized transplants, one-year survival remains at $100 \%$ [3••].

Significant progress has been made in the surgical technique for VCA, particularly for facial allografts. Cadaveric dissections and clinical cases have demonstrated that full facial allografts, including underlying segments of the craniofacial skeleton, can be supported on just the facial vessels, facilitating both efficient donor tissue retrieval and coaptation of facial nerve branches [15-17]. In addition, teams who have performed several face transplants have reported progressive reduction in intraoperative blood loss and shorter OR times [4•, 5••].

The surgical principles of hand transplantation are based primarily on upper extremity replantation, including the use of arm tourniquets to control bleeding [18]. Teams have modified their technique as they have gained experience. The Pittsburgh team has described the use of delayed arterial anastomosis. This procedure begins with osteosynthesis, followed by repair of extensor tendons, dorsal veins and nerves. Finally, the radial and ulnar arteries are anastomosed. This method has allowed precise repair of the median and ulnar nerves in a bloodless, non-congested field, and while ischemic time is undoubtedly prolonged, results to date suggest that this has not had a detrimental effect on outcome [19].

The initial cases of concomitant face and hand transplantation have raised significant surgical concerns due to prolonged operating times, in the region of $18-24 \mathrm{~h}$, and extensive blood transfusion requirements [3••, 20]. Both 
reported recipients of concomitant face and hand transplants received massive blood transfusions. Lantieri et al. [5*•] reported that the first recipient required transfusion of $66 \mathrm{U}$ of packed red blood cells. While less blood was needed for the second recipient, requirements were nonetheless considerable, with $20 \mathrm{U}$ of packed red blood cells being transfused $[6 \bullet \bullet]$. Such intra-operative blood loss may result in hemodynamic instability and further contribute to compromise of the allograft. In addition, the administration of massive blood transfusion in isolated face transplant recipients has been associated with complications including dilutional coagulopathy and rhabdomyolysis [21].

Although not a concomitant face and hand transplant, many of the potential risks described by Gordon et al. [4•] as being associated with multiple simultaneous VCAs were clearly demonstrated in a Turkish patient who underwent single stage, quadruple limb transplantation [22]. A detailed report in the medical literature is still awaited, but information released through the media indicates that this patient had a turbulent post operative course, necessitating re-amputation of all transplanted limbs, but despite this and aggressive resuscitative efforts, the patient died on postoperative day 3 [22].

Donor-related issues must also be considered. Teams are gaining ever more experience in coordinating the procurement of vascularized composite allografts with organ retrieval teams to avoid any disruption to the harvest of organs for life-sustaining transplants; clearly harvest of both face and upper extremity allografts for concomitant transplantation will require dedicated teams and refined procedures.

Restoration of donor integrity following removal of the face and the hands must not be overlooked. Current VCA teams have described well-accepted methods of restoring face transplant donors [23•]. However, it is possible that procurement of multiple VCAs will be less acceptable to donors and their families, which could potentially have a negative impact on VCA donation in general. Clearly restoring body integrity and appearance of the donor with both facial and upper extremity prostheses prior to return of the remains to the family is of high importance.

\section{Immunological Challenges}

To date, the majority of isolated face and hand transplants have been maintained successfully with conventional triple therapy immunosuppression including tacrolimus, myco-

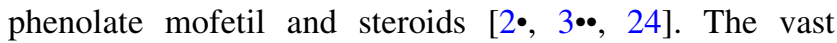
majority of both face and hand transplant recipients have experienced at least one episode of acute rejection, all of which have responded to treatment, most often with increased doses of immunosuppression or corticosteroid bolus $[3 \bullet \bullet, 8 \bullet$. In some cases, specifically in cases when rejection correlated with viral reactivation, extracorporeal photochemotherapy was used as an alternative in an effort to avoid increased immunosuppression, with encouraging results [25]. Interestingly, in contrast to the $100 \%$ graft survival enjoyed by isolated hand or face transplants, the incidence of acute rejection in both is higher than in solid organ transplantation [26]. The high prevalence of acute rejection episodes in vascularized composite allografts may reflect the immunogenicity of skin, but equally this apparent disparity may be confounded by the accessibility of VCAs, as visual inspection can reveal signs of rejection where solid organs require blood work or an invasive biopsy for a diagnosis. Frequent early diagnosis and treatment based on clinical skin changes in VCA is illustrated in the case of a face transplant recipient who was treated promptly for acute rejection, which was believed refractory until a history of recurrent rosacea in the donor came to light. Treatment with metronidazole cream resulted in complete resolution [10].

Chronic rejection, which is well described in solid organ transplantation and continues to have a very significant impact on long-term survival of organ allografts, has not yet been reported in face transplantation [27, 28]. One hand allograft loss, in which intimal hyperplasia was observed on histological examination, may have been attributable to chronic rejection, however other factors may have been involved and the etiology in this case is not clear [29]. Episodes of acute rejection have been identified as a risk factor for chronic rejection in organ transplantation, which makes the current lack of definite cases of chronic rejection following VCA an interesting area for further study [30, 31]. Long-term follow-up will be required to determine the functional and immunological effects of chronic rejection in face and hand transplantation, whether isolated or concomitant.

In addition to the immunological issues which must be addressed in all VCA recipients, concomitant face and hand transplantation may present unique challenges. The quantity of soft tissue involved in a combined procedure is significantly greater than either allograft in isolation [32•]. Skin is considered the most susceptible of all tissues to rejection, thus, it is possible that transplantation of such large volumes of skin may present a significant immunological burden [33].

Human recipients of large solid organ allografts have had favorable outcomes when compared to smaller transplants [34]. Large animal studies of kidney and cardiac transplantation have also demonstrated a possible beneficial effect of higher antigenic load on allograft survival [35]. Recent studies using small animal models of VCA 
have reported similar results [36]. While these findings may suggest a beneficial immunological effect from transplantation of larger amounts of donor tissue, further studies are required to confirm these reports and establish a mechanism for this effect, and even if true, any such benefit would have to be balanced against the other significant challenges presented by these complex procedures.

It has been reported that recipients of concomitant face and hand transplants have not required increased doses of immunosuppression to prevent graft rejection, and that at post mortem examination the French recipient's grafts were free from histological evidence of rejection [5••]. However, it is important to note that that there is not yet sufficient data available, nor experience in this area to make firm conclusions regarding the immunosuppressive requirements for concomitant face and hand transplantation.

\section{Functional Outcomes}

Functional outcome and recovery of both sensory and motor innervation following isolated face and hand transplantation have so far been encouraging [2•, 7, 37]. Pomahac et al. described return of mechanical and thermal sensation occurring as soon as three months after transplantation [6*0]. Other face transplant recipients have consistently recovered full sensation by 8 months postoperatively [5••, 38, 39]. Return of sensation has been reported even in the absence of adequate nerve repair; Lantieri et al. [6*0] described restoration of sensation when the mental nerves of the donor were placed near the mental foramen of the recipients without nerve coaptation in a case where insufficient length of recipient mental nerve remained to permit anastomosis. Sensory recovery in the distribution of the trigeminal nerve has also been described in the absence of proper nerve repair. The facial nerve was restored in these cases; the proposed explanation for sensory recovery is that interconnections formed between the motor facial nerve and the sensory trigeminal nerve [8•].

Overall, motor recovery has occurred more slowly than sensory re-innervation in facial transplantation. Lantieri et al. [40] described initial motor recovery as early as two months post-transplant with complete mouth closure seen at 8 months. Similarly, the first recipient of a face transplant was able to move her upper lip 3 months after the operation with complete labial contact achieved at 6 months and contraction of chin muscles regained after a year [12]. Other recipients have regained motor function, including restoration of facial mimetics, gradually over the first year after transplantation [10]. Interestingly, ongoing motor improvements have been observed over a period of several years [41]. Facial transplantation has generally resulted in significant functional improvements. In the immediate post-operative period, recipients have improved breathing and oral intake; other important functions including, smelling, smiling and speaking have steadily improved as motor and sensory recovery progresses. Siemionow et al. [39] also reported that their patient experienced a reduction in chronic pain following transplant, presumably as a result of excision of the scarred and contracted tissues left by her injury and subsequent reconstructive efforts.

Follow-up reports of hand transplant recipients have shown encouraging sensory recovery as well. The International Registry on Hand and Composite Tissue Transplantation reported return of protective sensation in all European hand transplant recipients $(\mathrm{n}=30)$ between September 1998 and July 2010 with at least 1 year followup [3••]. Tactile sensibility was restored in $90 \%$ of these recipients, with $84 \%$ developing discriminative sensibility $[3 \bullet \bullet$.

Motor recovery in hand transplant recipients typically has been observed commencing between 9 and 15 months following transplantation. Return of extrinsic muscle function, allowing grip and pinch activities, generally occurs earlier than intrinsic muscle function. Sensory and motor recovery in hand transplant recipients has conferred the ability to perform daily activities, including eating, driving, grasping objects, shaving, using the telephone and writing [3••]. Landin et al. described improved function following hand transplantation, as reported by patients themselves, using the disabilities of arm, shoulder and hand questionnaire [37]. As with facial transplantation, ongoing motor improvements in hand transplant recipients, including ability to perform fine tasks such as pick up ballbearings, have been observed more than 5 years after transplantation [3••, 42].

Despite the encouraging clinical outcomes observed in isolated hand and face transplant programs, concerns over the potential for functional recovery in concomitant face and hand transplantation have been raised [20]. In humans, the sensorimotor cortical representation of amputated segments is diminished following injury and replaced with increased representation from surrounding, unaffected areas [43]. Reversal of this cortical re-organization has been demonstrated following hand transplantation, with restoration of a normal pattern of upper extremity representation, even if transplantation occurs years after the initially injury and amputation [44]. However, the face and the hand are represented in adjacent areas of the sensorimotor cortex, and it has been shown that tactile sensitivity in a transplanted hand can be markedly diminished when the ipsilateral face is touched simultaneously [45]. This raises the concern that following concomitant transplantation, it is possible that the transplanted face and hand may compete for sensorimotor cortical representation, resulting 
in unpredictable recovery. Therefore, further experimental evidence is required to determine the likely functional outcomes in concomitant face and hand transplantation.

\section{Patient Selection}

To date, the face and hand transplant experience has identified patient selection as a key determinant of success. Failure of both face and hand transplants has resulted from patient noncompliance with post-operative immunosuppression protocols $[3 \bullet \bullet, 46]$. Some teams have published methods for assessment, selection and preparation of individual face or hand transplant recipients [47, 48]. However, no patient selection criteria for concomitant face and hand transplantation have been suggested thus far. Given the complexity of the procedure and the uncertainty of functional outcomes, it seems appropriate that potential recipients should undergo a particularly robust, highly individualized patient selection process. This should involve assessing quality of life, motivations for transplantation, expectations, coping strategies, social support and ability to comply with follow-up in addition to the full medical work up required for any complex surgical procedure.

Recipients selected for face and hand transplantation have included those affected by burns, ballistic trauma, neurofibromatosis and animal attacks. The extent of facial tissue replaced, both craniofacial skeleton and overlying soft tissue, has varied considerably. Both unilateral and bilateral hand transplants have been performed at different levels between the wrist and shoulder. It has been suggested that burn patients with extensive injury to face and hands would be ideal candidates for concomitant face and hand transplantation [20]. However, the first such recipient was a burn patient and the poor outcome in this case raises a number of concerns. The sharing of resistance patterns between the multi-drug resistant pseudomonas aeroguinosa infection diagnosed post transplant, and an earlier infection during his acute phase burn treatment suggests the possibility of re-activation of persisting organisms from this original infection upon immunosuppression [24]. Colonization of burn wounds is common, and the possibility of reactivation of indolent infections in this cohort is a cause for concern. Similarly, the use of cadaveric skin allografts during the acute treatment of burns may result in sensitization of potential recipients and reduce the likelihood of successfully identifying suitable VCA donors [5••]. Complex burn injuries undoubtedly present some of the greatest challenges for the reconstructive surgeon, and the potential benefits of concomitant VCA to the most severely injured of these patients are considerable. However, as described, these patients also present significant challenges, and some, particularly those with high levels of panel reactive antibody following extensive use of cadaveric allograft, may unfortunately be unsuitable candidates. We would suggest that evaluation of burn patients for concomitant face and hand transplantation should include an exhaustive review of their microbiologic history and close consultation with transplant infectious disease specialists.

\section{Future Directions}

Concomitant face and hand transplantation, like vascularized composite allotransplantation in general, is a lifeenhancing rather than life-saving procedure. Therefore, the basic ethical question is whether the risks of surgery and the burden of immunosuppression are outweighed by the potential benefits to the patient. The death of the first recipient of concomitant face and hand transplantation and the removal of the hands in the second case clearly highlight the dangers of this procedure. However, transplantation of the face and hands together does offer single stage restoration of upper limb and facial form and function, and consequently a potentially dramatic improvement in quality of life to a severely disabled and disfigured group of patients. Performing face and hand transplantation sequentially, from separate donors, could be considered as a potential alternative to concomitant transplantation. Precedent for sequential, multiple transplants can be found in the organ transplant literature, as it can for simultaneous multiple transplants and we believe both approaches are worthy of further consideration and study [49, 50]. Sequential transplant may offer benefits in terms of reduced operating time and blood loss at the time of initial surgery, however this would be offset against the risk inherent in an additional major surgical procedure, and the possibility that sensitization could occur to the first transplant leading to an accelerated immune response against the second. However, at this time insufficient data are available to quantify the relative risks of sequential and concomitant VCA.

Presently, little experimental evidence has been gathered on concomitant face and hand transplantation. Cadaveric analyses have demonstrated that the quantity of skin transferred in concomitant face and hand transplantation is significantly greater than either allograft in isolation [32-]. Further preclinical study of transplantation of large quantities of antigenic tissue will be useful in determining the immunologic impact of such cases, and may contribute to refinement of immunosuppressive regimens for these cases.

Donor-specific transplant tolerance has now been achieved in a number of clinical trials in kidney transplantation [51, 52]. Induction of tolerance of skin-bearing allografts remains a more difficult challenge, which has not 
yet been achieved clinically. The use of bone marrow transplantation in hand transplantation has facilitated maintenance of allografts on tacrolimus monotherapy, significantly reducing but not eliminating the immunosuppressive burden [53]. Induction of vascularized composite allograft tolerance has been achieved across MHC barriers in large animal models, but further development is required to translate these findings into a clinically applicable protocol [54]. These studies provide proof of concept for VCA tolerance, and, if successfully translated to clinical practice, hopefully can improve the risk benefit ratio for all VCA recipients, including future candidates for concomitant face and hand transplantation. However, the long term benefits of tolerance would only be relevant if the short term risks of sepsis and operative complications can be overcome.

\section{Conclusions}

The face and hands both play uniquely important roles in an individual's functional independence, psychological wellbeing and social interaction. Reconstruction of either following severe injury remains highly challenging, and results are often suboptimal. Concomitant face and hand transplantation offers single stage restoration of function and form to an unprecedented standard. The early experience in this field, sadly, has highlighted many difficulties and challenges, and much remains unknown, not least the potential for functional recovery following such cases. The successful outcomes of individual VCAs and simultaneous multiple organ transplants offer encouragement. Patients left severely disabled and disfigured by extensive craniofacial and upper extremity tissue loss would undoubtedly benefit considerably from a concomitant approach if the acute phase complications observed to date can be successfully overcome. Many of the outstanding questions, such as the immunologic impact of such extensive skin transplantation, are amenable to further study in preclinical models. Other questions however, such as those concerning cortical reintegration and functional outcomes will only be satisfactorily addressed by clinical trials and longer term follow-up. We encourage teams considering patients for concomitant face and hand transplantation to proceed cautiously, and would welcome continued candid reporting of outcomes and collaboration between VCA centers to facilitate progress and development in this challenging area.

Acknowledgments Dr. Leonard has received grant support from the American Society of Transplantation.

Disclosure No potential conflicts of interest relevant to this article were reported.

\section{References}

Papers of particular interest, published recently, have been highlighted as:

- Of importance

•- Of major importance

1. Shanmugarajah K, Hettiaratchy S, Butler PE. Facial transplantation. Curr Opin Otolaryngol Head Neck Surg. 2012;20(4): 291-7.

2. - Schneeberger S, Landin L, Jableki J, et al.: Achievements and challenges in composite tissue allotransplantation. Transpl Int. 2011;24(8):760-769. Review paper outlining the European experience of face and hand transplantation. Functional, aesthetic and immunological outcomes are discussed.

3. • Petruzzo P, Lanzetta M, Dubernard JM, et al. The international registry on hand and composite tissue transplantation. Transplantation. 2010;90(12):1590-94. A report from the International Registry reporting detailed follow-up information on vascularized composite allotransplant cases performed between September 1998 and July 2010. Functional and immunosuppressive outcomes are described.

4. - Gordon CR, Zor F, Cetrulo C Jr, et al. Concomitant face and hand transplantation: perfect solution or perfect storm? Ann Plast Surg. 2011;67(3):309-314. This review discusses surgical, medical, immunologic and rehabilitation issues relevant to concomitant face and hand transplantation.

5. • Lantieri L, Hivelin M, Audard V, et al.: Feasibility, reproducibility, risks and benefits of face transplantation: a prospective study of outcomes. Am J Transplant. 2011;11(2):367-78. This paper reports on the experience of the Lantieri group in detail on face transplantation and includes a report of the first concomitant face and upper extremity transplant.

6. • Pomahac B, Pribaz J, Eriksson E, et al. Three patients with full facial transplantation. N Engl J Med. 2012;366(8):715-22. A report of the technical aspects and initial follow-up of 3 recipients of face transplantation. Includes a report of the second case of concomitant face and upper extremity transplantation.

7. Shanmugarajah K, Hettiaratchy S, Clarke A, Butler PE. Clinical outcomes of facial transplantation: a review. Int J Surg. 2011;9(8):600-7.

8. - Siemionow M, Ozturk C. An update on facial transplantation cases performed between 2005 and 2010. Plast Reconstr Surg. 2011;128(6):707e-20e. Review paper overviewing the first 13 face transplants performed.

9. Singhal D, Pribaz JJ, Pomahac B. The Brigham and women's hospital face transplant program: a look back. Plast Reconstr Surg. 2012;129(1):81e-8e.

10. Pomahac B, Pribaz J, Eriksson E, et al. Restoration of facial form and function after severe disfigurement from burn injury by a composite facial allograft. Am J Transpl. 2011;11(2):386-93.

11. Cavadas PC, Ibanez J, Thione A. Surgical aspects of a lower face, mandible, and tongue allotransplantation. J Reconstr Microsurg. 2012;28(1):43-7.

12. Dubernard JM, Lengele B, Morelon E, et al. Outcomes 18 months after the first human partial face transplantation. N Engl J Med. 2007;357(24):2451-60.

13. Gordon CR, Siemionow M, Papay F, et al. The world's experience with facial transplantation: what have we learned thus far? Ann Plast Surg. 2009;63(5):572-78.

14. Gomez-Cia T, Sicilia-Castro D, Infante-Cossio P, et al. Second human facial allotransplantation to restore a severe defect following radical resection of bilateral massive plexiform neurofibromas. Plast Reconstr Surg. 2011;127(2):995-6. 
15. Pomahac B, Pribaz JJ, Bueno EM, et al. Novel surgical technique for full face transplantation. Plast Reconstr Surg. 2012;130(3): 549-55.

16. Pomahac B, Lengele B, Ridgway EB, et al. Vascular considerations in composite midfacial allotransplantation. Plast Reconstr Surg. 2010;125(2):517-22.

17. Pomahac B, Pribaz J. Facial composite tissue allograft. J Craniofac Surg. 2012;23(1):265-7.

18. Kleinert HE, Jablon M, Tsai TM. An overview of replantation and results of 347 replants in 245 patients. J Trauma. 1980;20(5): 390-8.

19. Azari KK, Imbriglia JE, Goitz RJ, et al. Technical aspects of the recipient operation in hand transplantation. J Reconstr Microsurg. 2012;28(1):27-34.

20. Siemionow MZ, Zor F, Gordon CR. Face, upper extremity, and concomitant transplantation: potential concerns and challenges ahead. Plast Reconstr Surg. 2010;126(1):308-15.

21. Hinojosa Perez R, Porras Lopez M, Escoresca-Ortega AM, et al. Severe rhabdomyolysis after allogeneic transplantation of facial structures: a case report. Transplant Proc. 2010;42(8):3081-82.

22. ABC News: Quadruple Limb Transplant Patient Dies. http:// abcnews.go.com/blogs/health/2012/02/28/quadruple-limb-transplantpatient-dies/. Accessed Aug 2012.

23. Quilichini J, Hivelin M, Benjoar MD, et al. Restoration of the donor after face graft procurement for allotransplantation: report on the technique and outcomes of seven cases. Plast Reconstr Surg. 2012;129(5):1105-11. Details the use of facial masks for the restoration of seven face transplant donors.

24. Leonard DA, Gordon CR, Sachs DH, Cetrulo CL Jr. Immunobiology of face transplantation. J Craniofac Surg. 2012;23(1):268-71.

25. Hivelin M, Siemionow M, Grimbert P. Lantieri: extracorporeal photopheresis: from solid organs to face transplantation. Transpl Immunol. 2009;21(3):117-28.

26. Gheorghian A, Schnitzler MA, Axelrod DA, et al. The implications of acute rejection and reduced allograft function on health care expenditures in contemporary US kidney transplantation. Transplantation. 2012;94(3):241-9.

27. Birnbaum LM, Lipman M, Paraskevas S, et al. Management of chronic allograft nephropathy: a systematic review. Clin J Am Soc Nephrol. 2009;4(4):860-5.

28. Taylor DO, Edwards LB, Boucek MM, et al. Registry of the International Society for Heart and Lung Transplantation: twentyfourth official heart transplant report-2007. J Heart Lung Transpl. 2007;26(8):769-81.

29. Kaufman CL, Blair B, Murphy E, Breidenbach WB. A new option for amputees: transplantation of the hand. J Rehabil Res Dev. 2009;46(3):395-404.

30. Basadonna GP, Matas AJ, Gillingham KJ, et al. Early versus late acute renal allograft rejection: impact on chronic rejection. Transplantation. 1993;55(5):993-5.

31. Matas AJ, Gillingham KJ, Payne WD, Najarian JS. The impact of an acute rejection episode on long-term renal allograft survival. Transplantation. 1994;57(6):857-9.

32. - Gordon CR, Zor F, Siemionow M. Skin area quantification in preparation for concomitant upper extremity and face transplantation: a cadaver study and literature review. Transplantation. 2011;91(9):1050-56. Describes a study examining the amount of skin that would be harvested in concomitant face and hand transplantation, based on cadaveric dissection.

33. Murray JE. Organ transplantation (skin, kidney, heart) and the plastic surgeon. Plast Reconstr Surg. 1971;47(5):425-31.

34. Sanchez-Fructuoso AI, Prats D, Marques M, et al. Does renal mass exert an independent effect on the determinants of antigendependent injury? Transplantation. 2001;71(3):381-6.

35. Yamada K, Mawulawde K, Menard MT, et al. Mechanisms of tolerance induction and prevention of cardiac allograft vasculopathy in miniature swine: the effect of augmentation of donor antigen load. J Thorac Cardiovasc Surg. 2000;119(4 Pt 1):709-19.

36. Ulusal BG, Ulusal AE, Wei FC, Lin CY. Allograft mass as a possible contributing factor to the skin transplant outcome. J Surg Res. 2010;161(2):321-7.

37. Landin L, Bonastre J, Casado-Sanchez C, et al. Outcomes with respect to disabilities of the upper limb after hand allograft transplantation: a systematic review. Transpl Int. 2012;25(4):424-32.

38. Barret JP, Gavalda J, Bueno J, et al. Full face transplant: the first case report. Ann Surg. 2011;254(2):252-6.

39. Siemionow M, Papay F, Alam D, et al. Near-total human face transplantation for a severely disfigured patient in the USA. Lancet. 2009;374(9685):203-9.

40. Lantieri L, Meningaud JP, Grimbert P, et al. Repair of the lower and middle parts of the face by composite tissue allotransplantation in a patient with massive plexiform neurofibroma: a 1-year follow-up study. Lancet. 2008;372(9639):639-45.

41. Petruzzo P, Testelin S, Kanitakis J, et al. First human face transplantation: 5 years outcomes. Transplantation. 2012;93(2): 236-40.

42. Breidenbach WC, Gonzales NR, Kaufman CL, et al. Outcomes of the first 2 American hand transplants at 8 and 6 years posttransplant. J Hand Surg Am. 2008;33(7):1039-47.

43. Ramachandran VS, Rogers-Ramachandran D, Stewart M. Perceptual correlates of massive cortical reorganization. Science. 1992;258(5085):1159-60.

44. Giraux P, Sirigu A, Schneider F, Dubernard JM. Cortical reorganization in motor cortex after graft of both hands. Nat Neurosci. 2001;4(7):691-2.

45. Farne A, Roy AC, Giraux P, Dubernard JM, Sirigu A. Face or hand, not both: perceptual correlates of reafferentation in a former amputee. Curr Biol. 2002;12(15):1342-6.

46. Hui-Chou HG, Nam AJ, Rodriguez ED. Clinical facial composite tissue allotransplantation: a review of the first four global experiences and future implications. Plast Reconstr Surg. 2010; 125(2):538-46.

47. Gordon CR, Siemionow M, Coffman K, et al. The Cleveland Clinic FACES Score: a preliminary assessment tool for identifying the optimal face transplant candidate. J Craniofac Surg. 2009;20(6):1969-74.

48. Kumnig M, Jowsey SG, Rumpold G, et al. The psychological assessment of candidates for reconstructive hand transplantation. Transpl Int. 2012;25(5):573-85.

49. Simpson N, Cho YW, Cicciarelli JC, et al. Comparison of renal allograft outcomes in combined liver-kidney transplantation versus subsequent kidney transplantation in liver transplant recipients: analysis of UNOS database. Transplantation. 2006; 82(10):1298-303.

50. Soh KS, Jeng LB, Yang HR, et al. Simultaneous liver and kidney transplantation from different living donors. Transplant Proc. 2012;44(2):509-11.

51. Kawai T, Cosimi AB, Spitzer TR, et al. HLA-mismatched renal transplantation without maintenance immunosuppression. N Engl J Med. 2008;358(4):353-61.

52. Leventhal $\mathrm{J}$, Abecassis $\mathrm{M}$, Miller $\mathrm{J}$, et al. Chimerism and tolerance without GVHD or engraftment syndrome in HLA-mismatched combined kidney and hematopoietic stem cell transplantation. Sci Transl Med. 2012;4(124):124-8.

53. Lee WPA, Brandacher G, Schneeberger S, et al. The Pittsburgh hand transplant program-early experience with a novel immunomodulatory protocol in two patients. In: Presented at the American Association of Plastic Surgeons meeting. San Antonio, TX; March 20-23, 2010.

54. Horner BM, Randolph MA, Duran-Struuck R, et al. Induction of tolerance to an allogeneic skin flap transplant in a preclinical large animal model. Transpl Proc. 2009;41(2):539-41. 